\title{
Devil Facial Tumor Disease
}

National Cancer Institute

\section{Source}

National Cancer Institute. Devil Facial T umor Disease. NCI Thesaurus. Code C136978.

A transmissible neoplastic disease that occurs in the Tasmanian devil. It is spread by biting and is characterized by tumors on the face and mouth. 